\title{
Oxidative stress in aging: stayin' alive?
}

\author{
Yuji Ikeno ${ }^{\mathrm{a},},{ }^{,}$Lisa C. Flores ${ }^{\mathrm{a}}$ \\ ${ }^{a}$ Barshop Institute for Longevity and Aging Studies, The University of Texas Health Science Center at San Antonio, TX, \\ USA.
}

\begin{abstract}
There is still ongoing controversy about the oxidative stress theory of aging, particularly in mammals, after a significant number of studies have been conducted to test this theory. Results generated from the studies strongly indicate that accumulation of oxidative damage alone does not play a significant role as an underlying mechanism of aging, which calls into question that significant modifications to the theory are required to understand the relationship between oxidative stress and aging. To examine the exact role of oxidative stress in aging and age-related diseases, our laboratory has been conducting studies with unique animal models: 1) mice overexpressing or down-regulating thioredoxin (Trx) in the cytosol (Trx1) or in mitochondria (Trx2); and 2) rats overexpressing $\mathrm{Cu} / \mathrm{Zn}$ superoxide dismutase (SOD). Results generated from these studies strongly indicate that: 1) changes in oxidative stress and redox state could play more important roles in age-related pathological changes, e.g., cancer and metabolic disorders; 2) redox regulation of signaling pathways could play more important roles in aging than accumulation of oxidative damages; 3 ) the potential benefit of changes in oxidative stress and redox state could be organ/tissue specific; 4) the roles of oxidative stress could vary in different stages of life (i.e., young versus old); and 5) synergetic effects of changes in oxidative stress in multiple cellular compartments may be required to have a significant impact on aging. Therefore, the studies with more careful approach would uncover the exact roles and pathophysiological consequences of oxidative stress during aging.

Keywords: Oxidative stress, aging, age-related diseases, cancer, obesity, healthspan
\end{abstract}

The free radical theory of aging, originally proposed by Dr. Denham Harman in the 1950s and later modified as the oxidative stress theory of aging, has been extensively studied over the past 60 years. There is substantial evidence that strongly supports this theory: 1) the levels of oxidative damage to macromolecules increase with age; 2) extended lifespan in various animal models is correlated to reduced oxidative damage and/or increased resistance to oxidative stress. In the $1990 \mathrm{~s}$, the effects of oxidative stress on lifespan were examined using mice that genetically altered various components of the antioxidant defense system to directly test the oxidative stress theory of aging. In theory, the increased resistance to oxidative stress by the overexpression of major antioxidant enzymes increases the lifespan of mice, while reduced resistance

* Corresponding author: Yuji Ikeno, M.D., Ph.D.

Mailing address: Barshop Institute for Longevity and Aging Studies, The University of Texas Health Science Center at San Antonio, 15355 Lambda Drive, San Antonio, TX 78245-3207, USA.

E-mail: ikeno@uthscsa.edu

Received: 11 June 2020 / Accepted: 14 June 2020 to oxidative stress by the down-regulation of major antioxidant enzymes shortens the lifespan of mice. However, the overexpression of major antioxidant enzymes did not extend lifespan except in MCAT mice, which overexpressed catalase in mitochondria; and the down-regulation of major antioxidant enzymes did not shorten lifespan in mice, except in $\mathrm{Cu} / \mathrm{ZnSOD}$-null mice. Thus, a majority of the data from mice with genetically altered levels of major antioxidant enzymes do not support the oxidative stress theory of aging, which seriously calls into question the exact roles of oxidative damage/stress on the aging process in mammals.

Our laboratory has been conducting research to test the exact roles of oxidative stress in aging and age-related diseases using unique animal models: 1) mice overexpressing or down-regulating thioredoxin (Trx) in the cytosol (Trx1) or in mitochondria ( $\operatorname{Trx} 2)$; and 2) rats overexpressing $\mathrm{Cu} /$ ZnSOD. We chose to examine the effects of Trx in aging because of its unique characteristics: Trx has ability to attenuate the level of oxidative stress and alter redoxsensitive signaling pathways. Thus, Trx could have more diverse effects on pathophysiology during aging other than altering the accumulation of oxidative damages. We also conducted an aging study with $\mathrm{Cu} / \mathrm{ZnSOD}$ rats to test the possible species differences, i.e., mouse vs. rats. 
The studies with Trx1 and Trx2 transgenic and knockout mice revealed that: 1) either Trx 1 or Trx 2 overexpression showed a slight extension of lifespan mainly in the earlier part of life with a slight acceleration of cancer development in the later part of life; 2) synergetic overexpression of Trx 1 and Trx 2 shortened lifespan with enhanced cancer development and changes in signaling pathways involved in tumor growth, which was contrary to initial expectations; and 3) the ongoing study with mice down-regulating both Trx 1 and Trx 2 demonstrates a slight extension (9-10\%) of lifespan in both males and females. The $\mathrm{Cu} /$ $\mathrm{ZnSOD}$ rats study showed that $\mathrm{Cu} / \mathrm{ZnSOD}$ overexpression in Sprague-Dawley (SD) rats extends lifespan while $\mathrm{Cu} /$ ZnSOD transgenic mice and F344 rats showed no changes in lifespan. Interestingly, the life-extension of $\mathrm{Cu} / \mathrm{ZnSOD}$ transgenic SD rats is associated with: 1) reduced age-related cancer; 2) attenuated kidney pathology; 3) enhanced insulin sensitivity; and 4) reduced senescence-associated beta-galactosidase positive cells in adipose tissues. These unexpected, but very intriguing observations strongly sug- gest: 1) changes in oxidative stress and redox state could play more important roles in age-related pathological changes, e.g., cancer and metabolic disorders, than aging per se; 2) redox regulation of signaling pathways could play more important roles in aging than accumulation of oxidative damages; 3 ) the potential benefit of changes in oxidative stress and redox state could be organ/tissue specific; 4) the roles of oxidative stress could vary in different stages of life (i.e., young vs. old); and 5) synergetic effects of changes in oxidative stress in multiple cellular compartments, e.g., cytosol and mitochondria, may be required to have a significant impact on aging in mammals. We believe that oxidative stress plays important roles in aging in multiple ways. The detailed analyses of the roles of oxidative stress/cellular redox state in healthspan, agerelated diseases, organ specific functional changes, and different stages of life, will allow us to have a clearer picture and lead to a major paradigm shift of the oxidative stress theory of aging.

Cite this article as: Ikeno Y, Flores L C. Oxidative stress in aging: stayin'alive?[J]. Aging Pathobiology and Therapeutics, 2020, 2(2): 62-63. 